\title{
Increase in the Diagnosis and Severity of Presentation of Pediatric Type 1 and Type 2 Diabetes during the COVID-19 Pandemic
}

\author{
Brynn E. Marks ${ }^{a, b} \quad$ Aneka Khilnani ${ }^{b} \quad$ Abby Meyers $^{a, b} \quad$ Myrto E. Flokas $^{a}$ \\ Jiaxiang Gai ${ }^{c}$ Maureen Monaghan ${ }^{b, d}$ Randi Streisand ${ }^{b, d}$ Elizabeth Estrada ${ }^{a}$, \\ ${ }^{a}$ Division of Endocrinology, Children's National Hospital, Washington, DC, USA; ${ }^{b}$ George Washington University \\ School of Medicine and Health Sciences, Washington, DC, USA; 'Division of Biostatistics, Children's National \\ Hospital, Washington, DC, USA; 'Division of Psychology and Behavioral Health, Children's National Hospital, \\ Washington, DC, USA
}

\section{Keywords}

Pediatric type 1 diabetes · Pediatric type 2 diabetes .

Diabetic ketoacidosis - Hyperosmolar diabetic ketoacidosis . COVID-19

\begin{abstract}
Introduction: The impact of the COVID-19 pandemic on the incidence of pediatric type 1 (T1D) and type 2 diabetes (T2D) and severity of presentation at diagnosis is unclear. Methods: A retrospective comparison of 737 youth diagnosed with T1D and T2D during the initial 12 months of the COVID-19 pandemic and in the preceding 2 years was conducted at a pediatric tertiary care center. Results: Incident cases of T1D rose from 152 to 158 in the 2 years before the pandemic (3.9\% increase) to 182 cases during the pandemic (15.2\% increase). The prevalence of diabetic ketoacidosis (DKA) at T1D diagnosis increased over 3 years (41.4\%, 51.9\%, and $57.7 \%, p=0.003)$; severe DKA increased during the pandemic as compared to the 2 years before ( $16.8 \%$ vs. $28 \%, p=$ $0.004)$. Although there was no difference in the mean hemoglobin A1c (HbA1c) between racial and ethnic groups at T1D diagnosis in the 2-years pre-pandemic ( $p=0.31)$, during the pandemic HbA1c at T1D diagnosis was higher in non-Hispanic Black (NHB) youth $(11.3 \pm 1.4 \%$, non-Hispanic White
\end{abstract}

karger@karger.com

(c) 2021 S. Karger AG, Basel

www.karger.com/hrp

Karger"
$10.5 \pm 1.6 \%$, Latinx $10.8 \pm 1.5 \%, p=0.01)$. Incident cases of T2D decreased from 54 to 50 cases ( $7.4 \%$ decrease) over the 2 -years pre-pandemic and increased $182 \%$ during the pandemic $(n=141,1.45$ cases/month, $p<0.001)$. As compared to the 2-years pre-pandemic, cases increased most among $\mathrm{NHB}$ youth $(56.7 \%$ vs. $76.6 \%, p=0.001)$ and males $(40.4 \%$ vs. $58.9 \%, p=0.005)$. Cases of DKA ( $5.8 \%$ vs. $23.4 \%, p<0.001)$ and hyperosmolar DKA (0 vs. $9.2 \%, p=0.001$ ) increased among youth with T2D during the pandemic. Conclusions: During the pandemic, the incidence and severity of presentation of T1D increased modestly, while incident cases of T2D increased $182 \%$, with a nearly 6 -fold increase in DKA and nearly a $10 \%$ incidence of hyperosmolar DKA. NHB youth were disproportionately impacted, raising concern about worsening of pre-existing health disparities during and after the pandemic.

C 2021 S. Karger AG, Basel

\section{Introduction}

The World Health Organization declared the COVID-19 pandemic on March 11, 2020. While the effects of COVID-19 on diabetes-related outcomes have been extensively studied in adults, data about the incidence 
and severity of presentation of pediatric new-onset type 1 (T1D) and type 2 diabetes (T2D) are limited. In the early months of the pandemic, studies showed increasing rates of severe diabetic ketoacidosis (DKA) at diagnosis of T1D [1-4]. However, findings about the impact of the pandemic on the overall incidence of T1D yielded conflicting results $[1,2,4]$. Data assessing changes in the incidence and severity of presentation of pediatric T2D during the pandemic are more limited [5]. Many different factors may have led to changes in the incidence and severity of pediatric diabetes during the pandemic, including direct diabetogenic effects of the severe acute respiratory syndrome coronavirus 2 (SARS-CoV-2) [6], weight gain resulting from unintended consequences of social distancing measures [7], COVID-19-related stressors which may predispose to autoimmunity and insulin resistance $[8,9]$, and decreased primary care visits that may have led to a delay in the recognition and diagnosis of new-onset diabetes $[10,11]$.

The aim of this retrospective study of patients at a highly diverse pediatric tertiary care center was to compare differences in the following aspects of new-onset T1D and T2D during the initial 12 months of the COVID-19 pandemic: (1) cases of new-onset diabetes, (2) severity of presentation, and (3) differences in demographic and anthropometric characteristics. We hypothesized that cases of pediatric T1D did not change significantly during the pandemic and that cases of T2D increased, while the severity of presentation increased in both groups.

\section{Materials and Methods}

\section{Data Collection}

A retrospective review of the electronic medical record (EMR) identified youth diagnosed with T1D and T2D during the first year of the COVID-19 pandemic (March 11, 2020, to March 10, 2021) and in the time-matched periods from 2018 to 2019 and 2019 to 2020 at a pediatric tertiary care center in Washington, D.C. This study protocol was reviewed and deemed exempt by institutional review board at the Children's National Hospital.

Children's National Hospital is a 323-bed acute care hospital with 16 specialty care centers throughout the Washington, DC, metro area and is the only free-standing pediatric hospital between Philadelphia, Pittsburgh, Norfolk, and Atlanta. The Diabetes Program provides clinical care to approximately 1,800 youth with T1D and 600 youth with T2D annually.

All youth $\leq 21$ years of age with newly diagnosed T1D and T2D over this 3 year period were captured using ICD-10 diagnosis codes (E10.9, E11.9) and diabetes education logs. At our center, all youth with new-onset T1D are admitted, whereas youth with suspected T2D are only admitted for severe hyperglycemia, keto- sis, challenging social circumstances, or an inability to schedule urgent outpatient follow-up. Demographic data were extracted from the EMR along with anthropometric measures at diagnosis. The initial plasma glucose, venous $\mathrm{pH}$, serum bicarbonate, and hemoglobin A1c (HbA1c) at presentation are reported. Weight and BMI data from 6 to 18 months before T2D diagnosis were gathered for youth diagnosed between 3/11/2019 and 3/10/2021. COVID-19 status at diagnosis was assessed using SARS-CoV-2 PCR results from a molecular diagnostic assay performed on a nasopharyngeal swab (ePlex SARS-CoV-2 Test [GenMark Diagnostics], Simplexa COVID-19 assay [DiaSorin Molecular LLC], and Xpert Xpress SARS-CoV-2 [Cepheid]). During the period from March 15, 2020, to April 12, $2020(n=14)$, only symptomatic patients were tested for SARS-CoV-2. Universal screening for inpatients and those seen in the emergency department was instituted on April 13, 2020; all patients admitted between April 13, 2020 , and March 10, 2021, had PCR testing $(n=304)$. Outpatients $(n=56)$ were not tested for SARS-CoV-2. Pairwise deletion was used for missing data.

\section{Diagnosis of T1D and T2D}

EMR data were reviewed to confirm the diagnosis of new-onset diabetes, type of diabetes, and severity of presentation. Diabetes was defined according to American Diabetes Association criteria: fasting plasma glucose $\geq 126 \mathrm{mg} / \mathrm{dL}, \mathrm{HbA1c} \geq 6.5 \%$ (48 mmol/ $\mathrm{mol}$ ), or a random blood glucose $\geq 200 \mathrm{mg} / \mathrm{dL}$ with symptoms of hyperglycemia [12].

T1D was diagnosed in children who were not overweight (BMI $Z$-score $<1.04$ ) [13] and/or those with at least 2-positive autoantibodies (GAD65, islet antigen 2 [IA2], insulin, zinc transporter 8 , and islet-cell antibody screen) and/or age $<8$ years. T2D was diagnosed if obese (BMI Z-score >1.64) [13] in the absence of DKA and pancreatic autoantibodies. All youth with overlapping features were further categorized based on the clinical impression of a pediatric endocrinologist (B.E.M.) formed from review of data at initial diagnosis and in follow-up, including family history, insulin requirements, and weight trajectories over time. Youth with positive genetic testing or strong clinical suspicion for monogenic diabetes were excluded $(n=7)$.

\section{Definitions of DKA, HHS, and Hyperosmolar DKA}

DKA was defined by plasma glucose $>200 \mathrm{mg} / \mathrm{dL}$, venous $\mathrm{pH}$ $<7.3$ or serum bicarbonate $<15 \mathrm{mmol} / \mathrm{L}$, and ketonemia or ketonuria [14]. DKA severity was defined as mild ( $\mathrm{pH}<7.3$ or bicarbonate $<15 \mathrm{mmol} / \mathrm{L}$ ), moderate $(\mathrm{pH}<7.2$ or bicarbonate $<10$ $\mathrm{mmol} / \mathrm{L})$, and severe $(\mathrm{pH}<7.1$ or bicarbonate $<5 \mathrm{mmol} / \mathrm{L})$ [14]. Hyperglycemic hyperosmolar syndrome (HHS) was defined by plasma glucose $>600 \mathrm{mg} / \mathrm{dL}$, effective serum osmolality $>330$ $\mathrm{mOsm} / \mathrm{kg}$, and $\mathrm{pH}>7.25$ or serum bicarbonate $>15 \mathrm{mmol} / \mathrm{L}$, whereas hyperosmolar DKA was defined by the same glucose and plasma osmolality values with a $\mathrm{pH} \leq 7.25$ or bicarbonate $\leq 15$ $\mathrm{mmol} / \mathrm{L}$ [15].

\section{Categorizing Obesity Severity}

Obesity was defined as follows: Class 1 (BMI $\geq 95$ th percentile for age and sex), Class 2 (BMI $\geq 120 \%$ of the 95 th percentile for age and sex or BMI $\geq 35 \mathrm{~kg} / \mathrm{m}^{2}$, whichever is lower), and Class 3 (BMI $\geq 140 \%$ of the 95 th percentile for age and sex or BMI $\geq 40 \mathrm{~kg} / \mathrm{m}^{2}$, whichever is lower) $[16,17]$. 
Table 1. Demographic characteristics at the time of presentation for youth newly diagnosed with T1D before and during the COVID-19 pandemic ${ }^{a}$

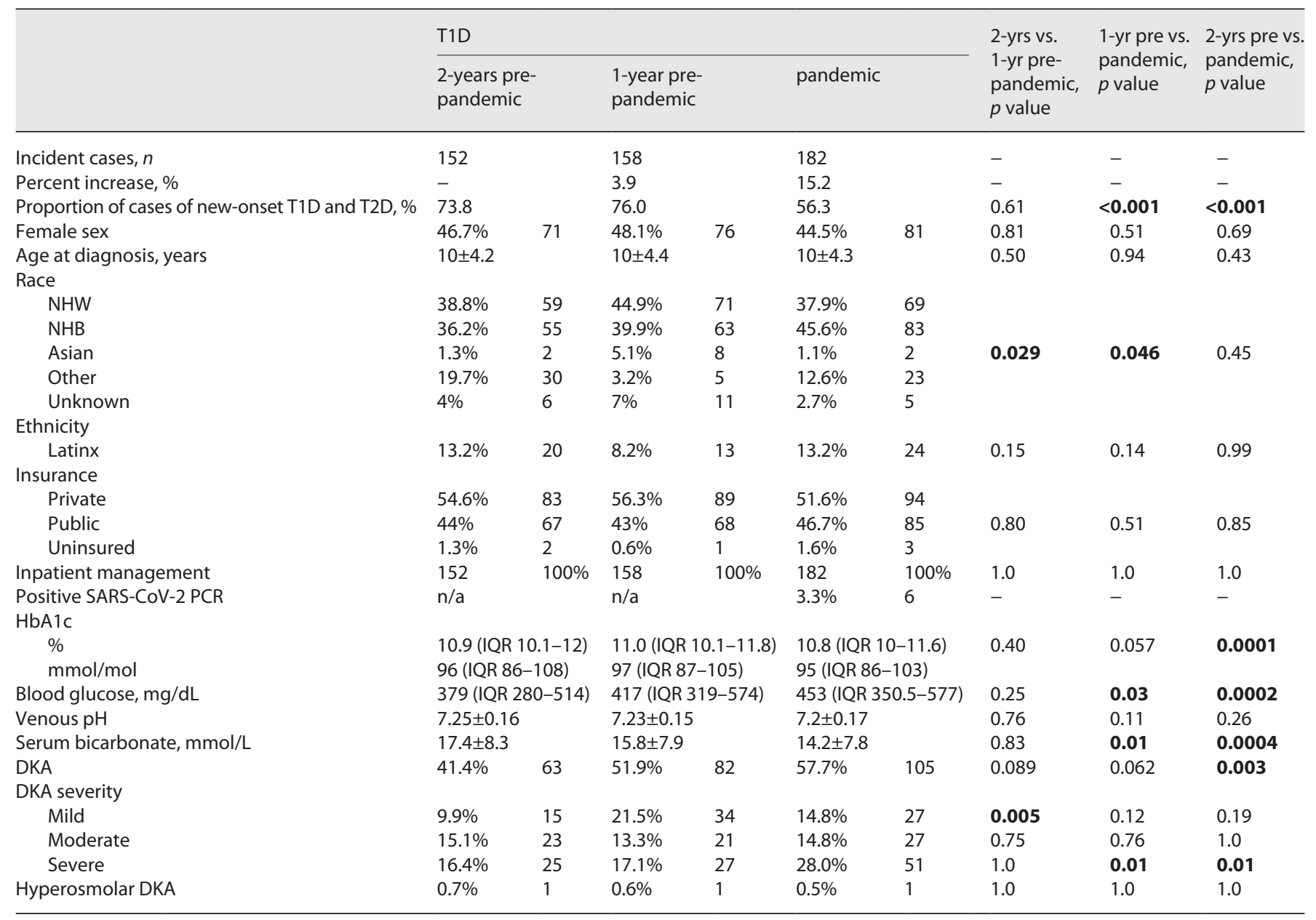

T1D, type 1 diabetes; DKA, diabetic ketoacidosis; HbA1c, hemoglobin A1c; NHW, non-Hispanic White; NHB, non-Hispanic Black; SARS, severe acute respiratory syndrome coronavirus. ${ }^{\text {a }}$ Normally distributed, continuous variables reported as mean \pm standard deviation. Median and interquartile range reported for skewed data.

Statistical Analyses

IBM SPSS Statistics for Windows version 26.0 (IBM, Armonk, NY, USA) and SAS 9.4 (SAS Institute, Cary, NC, USA) were used for analyses. Two-tailed $p$ values $\leq 0.05$ were considered significant. Characteristics from the study sample are stratified by type of diabetes and year of diagnosis (2-years pre-pandemic, 1-year pre-pandemic, and during the pandemic). Some analyses are further stratified into the 3 most common racial and/or ethnic groups in our population, consistent with prior publications: non-Hispanic White (NHW), non-Hispanic Black (NHB), and Latinx [18, 19]. Descriptive categorical data are reported as frequency and proportion; continuous variables are reported as means and standard deviations or medians and interquartile ranges, as appropriate. $\chi^{2}$, Fisher's exact, independent samples $t$ tests, ANOVA, and Mann-Whitney tests were used to evaluate differences between the populations diagnosed during this 3-year period and between different racial and ethnic groups. Cochran-Armitage trend tests were used to assess for the increases across the 3-year time period. A linear regression model was fitted to assess trends in the number of cases of T2D in each of the 3 years. The slope of each regression line was tested to see if it was significantly different from zero, and the 3 slopes were compared by including the interaction term.

\section{Results}

A total of 737 youth were diagnosed with T1D $(n=$ $492)$ and T2D $(n=245)$ in the 3 -year period. The demographic characteristics of youth with T1D versus those with T2D can be summarized as follows: NHW, $40.4 \%$ versus $4.9 \%(p<0.001)$; NHB, $40.9 \%$ versus $68.2 \%(p<$ $0.001)$; Latinx, $11.6 \%$ versus $21.2 \%(p=0.001)$; and pub- 


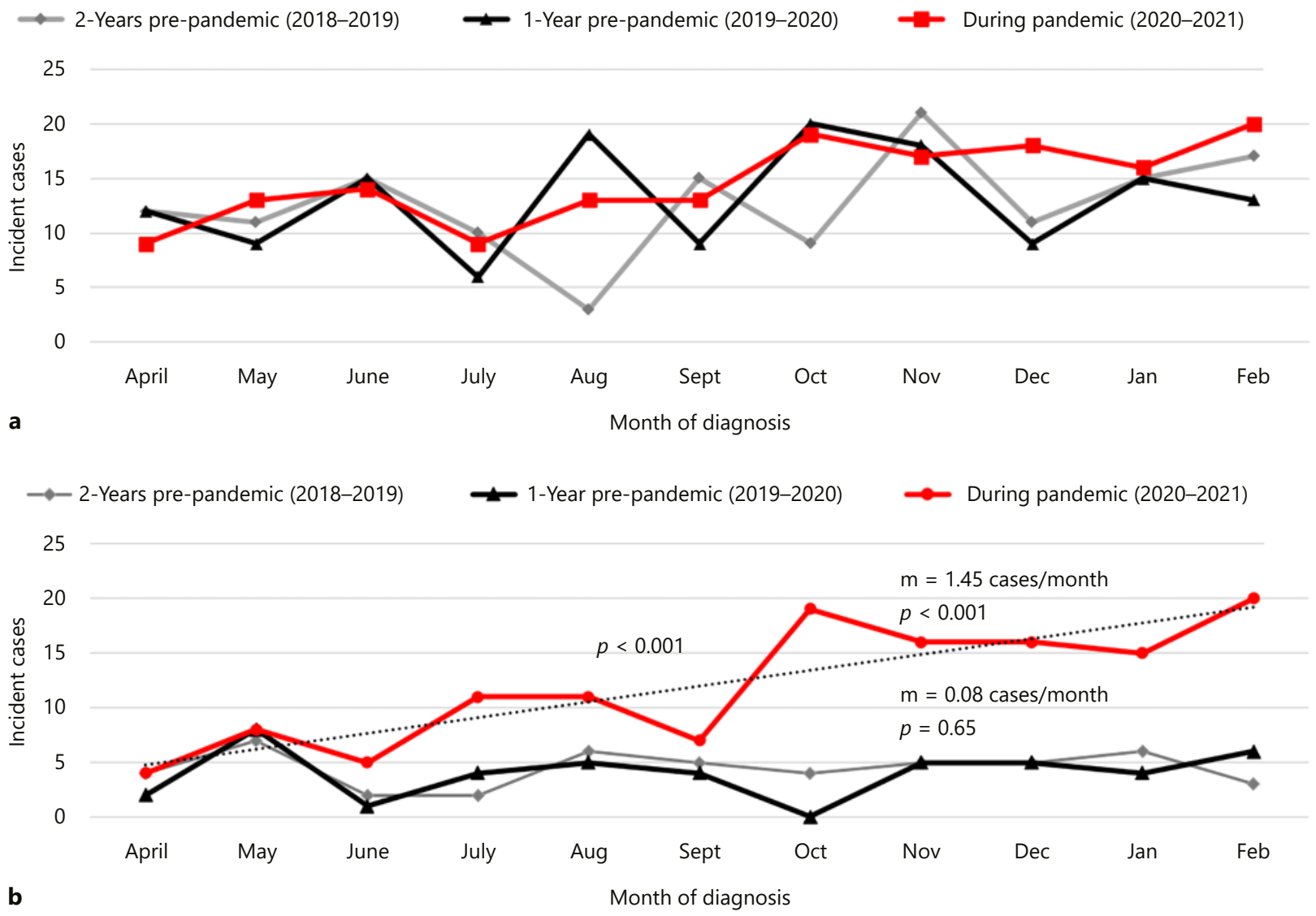

Fig. 1. Incident cases of new onset type 1 diabetes (a) and type 2 diabetes $(\mathbf{b})$ in the 2 -years before and during the first 12-months of the COVID-19 pandemic. Trendlines for the pandemic year and the average number of cases per month in the 2 years pre-pandemic are depicted.

licly insured $44.7 \%$ versus $81.6 \%(p<0.001)$. T2D accounted for $26.2 \%(n=54 / 206)$ and $24.0 \%(n=50 / 208)$ ( $p=0.61)$ of all incident cases of diabetes in the 2-years before the pandemic, rising to $43.7 \%(n=141 / 323)$ during the pandemic $(p<0.001)$. SARS-CoV-2 testing was performed in $76.4 \%$ ( $n=247 / 323)$ of youth diagnosed during the pandemic; $3.3 \%$ of all youth with $\mathrm{T} 1 \mathrm{D}$ and $3.5 \%$ with T2D tested SARS-CoV-2 positive $(p=1)$.

\section{Type 1 Diabetes}

Incident cases of T1D rose from 152 to 158 in the 2 years before the pandemic ( $3.9 \%$ increase) to 182 cases during the pandemic (15.2\% increase). Further details about the demographic characteristics and severity of presentation are shown in Table 1. There was an association between race/ethnicity and year before the pre-pan- demic versus the pandemic period $(p=0.046)$, with a trend toward an increasing number of cases among NHB youth over the 3 -year period $(p=0.08)$. There was no significant change in the number of Latinx or publicly insured youth diagnosed with T1D during these 3 years.

There was no statistically significant difference in the percentage of youth presenting in DKA 1 and 2 years before the pandemic $(p=0.065)$ or the year before the pandemic versus the pandemic year $(p=0.28)$; however, rates of DKA increased over the 3 years $(p=0.003)$. Although there was no significant difference in the percentage of youth presenting in severe DKA in the 2-years pre-pandemic $(p=1)$, the prevalence of severe DKA was higher during the pandemic $(28.0 \%)$ than the average rate in the 2 prior years $(16.8 \%, p=0.004)$ (Fig. 1$)$. There was no significant difference in the rates of DKA among $\mathrm{NHB}$ 
Table 2. Demographic characteristics at the time of presentation for youth newly diagnosed with T2D before and during the COVID-19 pandemic ${ }^{a}$

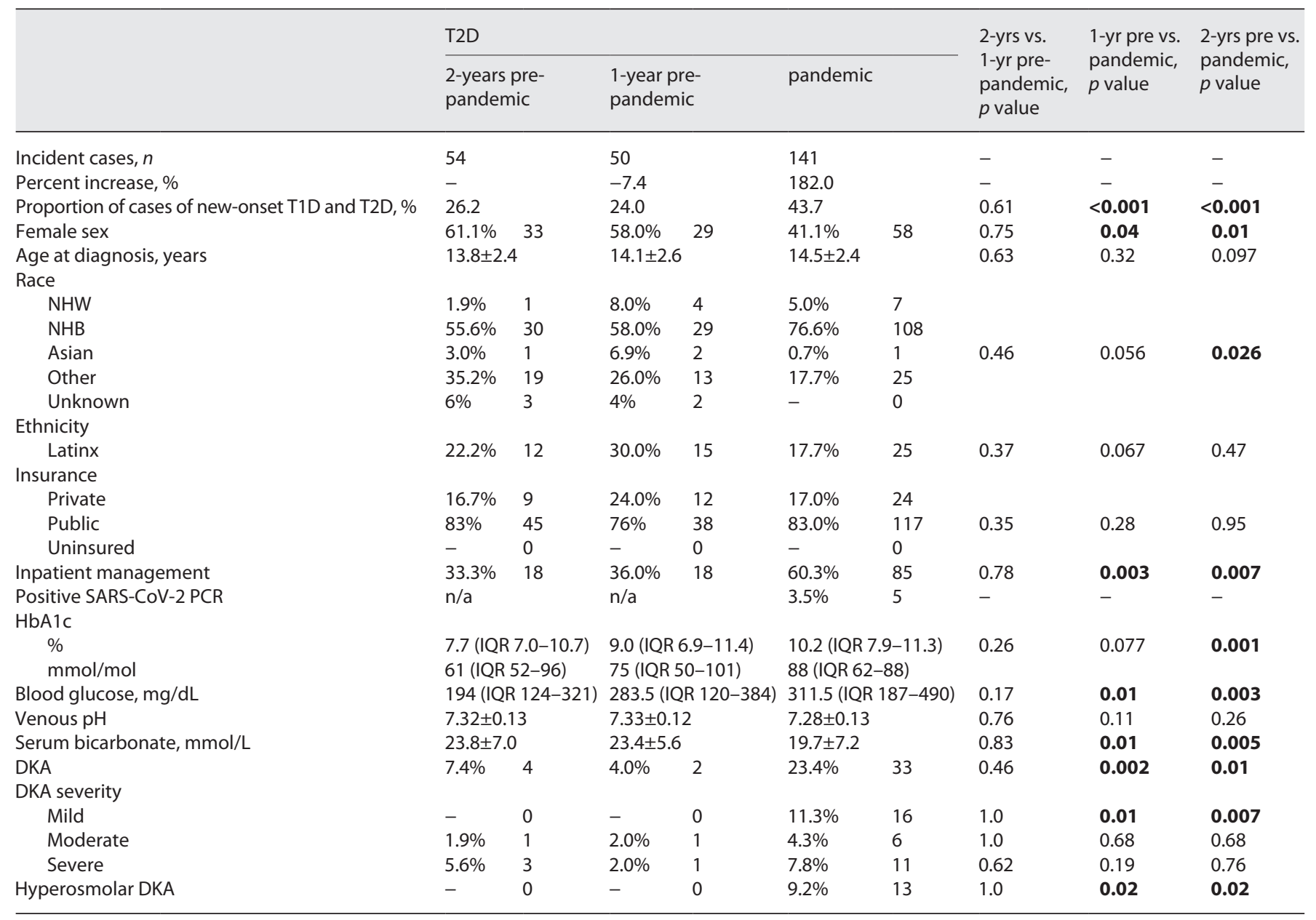

T2D, type 2 diabetes; DKA, diabetic ketoacidosis; HbA1c, hemoglobin A1c; NHW, non-Hispanic White; NHB, non-Hispanic Black; SARS, severe acute re-

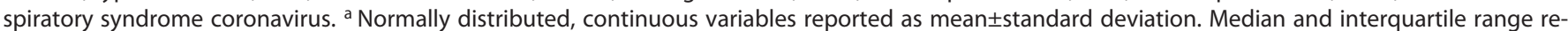
ported for skewed data.

(62.7\%), NHW (55.1\%), and Latinx (50\%, $p=0.45)$ youth during the pandemic; however, the proportion of NHB youth presenting in DKA during the pandemic $(62.7 \%)$ was greater than that in the preceding 2 years $(45.8 \%, p=$ 0.02). Although there was no difference in $\mathrm{HbAlc}$ according to race/ethnicity among youth diagnosed in the 2 prepandemic years $(p=0.31)$, there were between group differences during the pandemic ( $p=0.01)$; post hoc analyses revealed a higher mean $\mathrm{HbAlc}$ at diagnosis among NHB youth $(11.3 \% \pm 1.4 \%,[100 \pm 15 \mathrm{mmol} / \mathrm{mol}])$ as compared to NHW youth $(10.5 \pm 1.6,[92 \pm 18 \mathrm{mmol} /$ mol]) $(p=0.008)$. The mean HbAlc at diagnosis during the pandemic as compared to the 2 years before decreased nonsignificantly among NHW $(10.7 \pm 1.8 \%$ [94 \pm 20 $\mathrm{mmol} / \mathrm{mol}]$ vs. $10.5 \pm 1.6 \%[92 \pm 18 \mathrm{mmol} / \mathrm{mol}], p=0.42)$ and Latinx youth $(11.2 \pm 1.4 \%[99 \pm 15 \mathrm{mmol} / \mathrm{mol}] \mathrm{vs}$. $10.8 \pm 1.5 \%[94 \pm 17 \mathrm{mmol} / \mathrm{mol}], p=0.3)$ and increased nonsignificantly among NHB youth $(11.2 \pm 1.6 \%$ [ $98 \pm 17$ $\mathrm{mmol} / \mathrm{mol}]$ vs. $11.3 \pm 1.4 \%[100 \pm 15 \mathrm{mmol} / \mathrm{mol}], p=$ $0.49)$.

\section{Type 2 Diabetes}

Cases of new-onset T2D declined by 7.4\% from 2 -years pre-pandemic $(n=54)$ to 1 -year pre-pandemic $(n=50)$ and then increased $182 \%$ during the pandemic $(n=141)$. Further details about the demographic characteristics and severity of presentation are shown in Table 2. Sixtyeight percent of youth diagnosed with T2D during the 


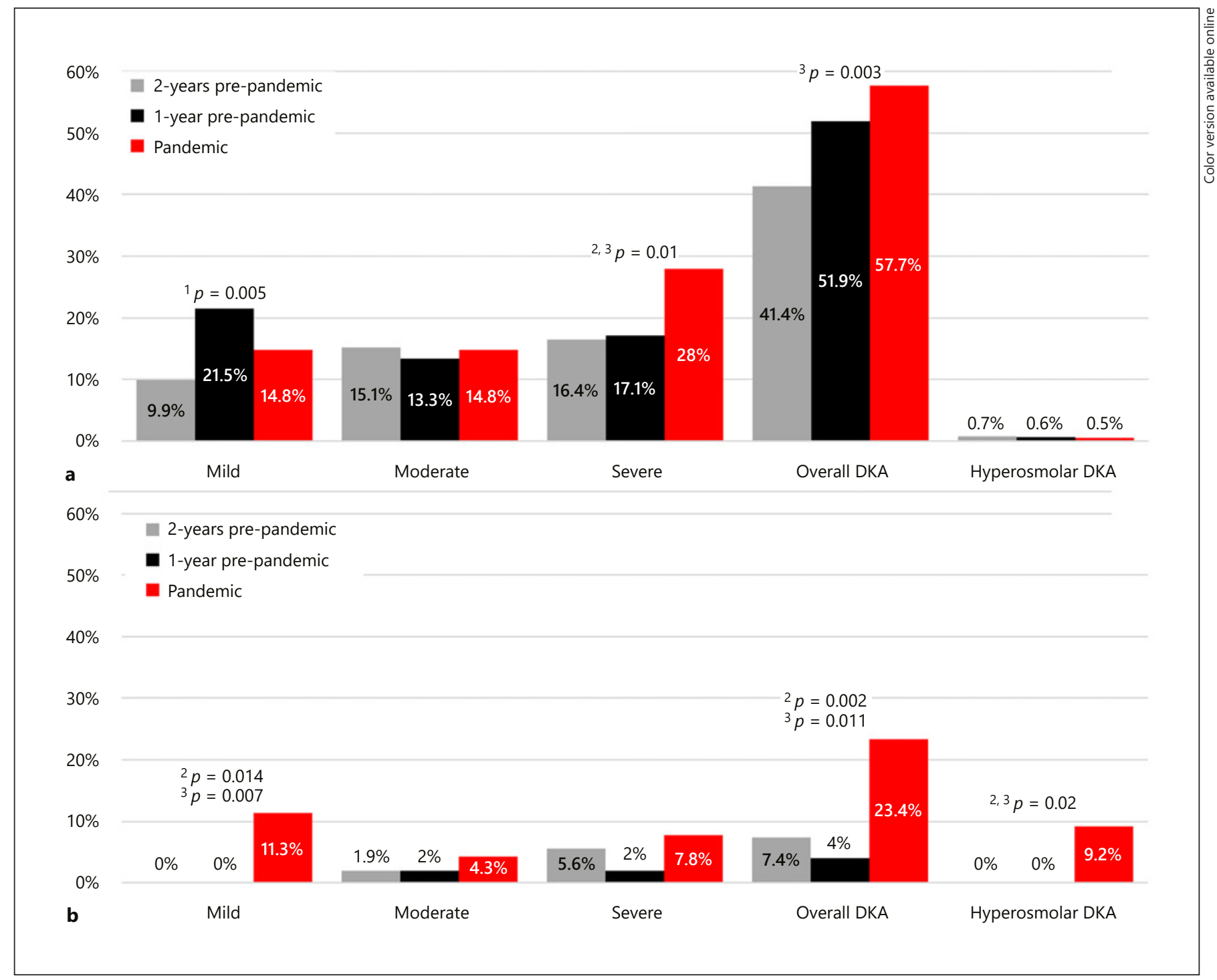

Fig. 2. The percentage of youth with newly diagnosed type 1 diabetes (a) and type 2 diabetes (b) presenting in DKA and hyperosmolar DKA in the 2-years before and during the first 12-months of the COVID-19 pandemic. ${ }^{1}$ Denotes a significant difference between 1 - and 2 -years pre-pandemic. ${ }^{2}$ Denotes a significant difference between 1-year pre-pandemic vs the pandemic year. ${ }^{3}$ Denotes a significant difference between 2 -years pre-pandemic vs the pandemic year.

3-year period were NHB and $81.6 \%$ were publicly insured. Despite no significant change in the proportion of cases among NHB youth in the 2 -years pre-pandemic $(p=0.81)$, cases increased during the pandemic as compared to the year before $(p=0.01)$; cases among NHB youth increased by $272 \%$ during the pandemic. There was no significant increase in cases among NHW youth $(p=$ $0.79)$ or Latinx youth $(p=0.32)$ over these same intervals. There was no change in the sex predominance of youth diagnosed with T2D in the 2-years pre-pandemic (38.9\% and $42 \%$ male, $p=0.75$ ), but during the pandemic as compared to the 2-years prior, more youth diagnosed with T2D were male $(58.9 \%, p=0.005)$. Whereas there was no variation in the number of cases of T2D per month in each of the 2 years before the pandemic $(p=0.77)$, rates of increase were greater during the pandemic $(p<0.001)$, and cases increased steadily by 1.45 cases/month throughout the pandemic $(p<0.001)$ (Fig. 2$)$.

The prevalence of DKA in T2D did not change significantly over the 2-years pre-pandemic $(p=0.46)$ but 
increased from 1-year pre-pandemic (4.0\%) to the year of the pandemic $(23.4 \%, p=0.002)$ (Fig. 1). Increases in DKA were driven by increase in mild DKA ( 0 cases in the 2 -years pre-pandemic vs. $11.3 \%$ during the pandemic, $p=0.001)$ without any significant increase in moderate $(p=0.67)$ or severe DKA $(p=0.76)$. NHB youth accounted for $92.3 \%$ of the cases of DKA throughout the 3-year period; $100 \%$ of those presenting in DKA were publicly insured. The median glucose at presentation $(311.5 \mathrm{mg} /$ dL vs. $283.5 \mathrm{mg} / \mathrm{dL}, p=0.03)$ and HbAlc values $(10 \pm$ $2.3 \%$ vs. $9.3 \pm 2.5 \%, p=0.001$ ) were higher during the pandemic but did not increase significantly between 1and 2-years pre-pandemic. There were no cases of HHS during the study period. Cases of hyperosmolar DKA rose from none in the 2-years pre-pandemic to $13(9.2 \%$, $p=0.02$ ) during the pandemic. Twelve of the 13 cases (92.3\%) of hyperosmolar DKA occurred in NHB youth who were publicly insured. The percentage of youth requiring inpatient management at diagnosis increased from $34.6 \%$ in the 2 -years pre-pandemic combined to $60.3 \%$ during the pandemic $(p<0.001)$.

Weight data at the time of diagnosis of T2D were available for $94 \%$ of youth 1 -year pre-pandemic $(n=47 / 50)$ versus $94.3 \%$ during the pandemic $(n=133 / 141)$. BMI at diagnosis was available for $92 \%$ and $88.7 \%$ of youth, respectively. There were no differences in weight or BMI at diagnosis before versus during the pandemic. At the time of diagnosis, $73.7 \%$ of youth with available BMI data had class $2(29.8 \%, n=51)$ or 3 obesity $(43.9 \%, n=75)$. There was a trend toward an association between the pandemic and obesity class $(p=0.08)$; however, post hoc analyses failed to show significant changes in the individual classes over time. There was no association between obesity class and race/ethnicity. Measurements 6-18 months before diagnosis were available for 56 youth (29.3\%) at an average interval of $0.91 \pm 0.35$ years before diagnosis. Fifteen $(26.8 \%)$ experienced weight loss in the time leading up to diagnosis. Among those who gained weight prior to diagnosis, weight increased by a median of $11.3 \mathrm{~kg}$ (IQR $6.1-17.7 \mathrm{~kg}$ ) or $13.0 \%$ (IQR $6.9 \%-19.1 \%$ ) of their initial body weight.

\section{Discussion}

Despite no significant change in the number of cases of pediatric T2D or severity of presentation over the 2 years before the pandemic, we found a $182 \%$ increase in pediatric T2D with a steady rate of monthly increase during the first 12 months of the COVID-19 pandemic. This increase in cases was accompanied by a nearly 6 -fold rise in DKA and a 9.2\% incidence of hyperosmolar DKA. In the 2 years before the pandemic, cases of T2D accounted for $25.1 \%$ of all newly diagnosed diabetes at our center as compared to $43.7 \%$ during the pandemic. These findings are a significant departure from prior studies indicating that T2D accounted for $11 \%$ of all cases of pediatric diabetes with a relative annual increase in cases of $4.8 \%$ per year $[20,21]$. Cases of T1D increased by $3.9 \%$ from $1-$ to 2 -years pre-pandemic and rose by $15.2 \%$ during the first 12 months of the pandemic accompanied by a more than $10 \%$ absolute percentage point increase in the incidence of severe DKA. The increase in cases of diabetes during the pandemic is particularly notable given the decline in the number of unique patients seen at our center during that time. Annual reports indicate that $\mathrm{CNH}$ provided care to 237,047 and 240,390 unique patients in the 2018 and 2019 fiscal years, respectively, compared to 223,856 in the 2020 fiscal year. The rise in cases of T2D and increased severity of presentation of both T1D and T2D during the pandemic disproportionately impacted $\mathrm{NHB}$ youth.

Given these trends over time, there was an undeniable increase in incident cases of $\mathrm{T} 2 \mathrm{D}$ during the pandemic; however, it is more difficult to ascertain the significance of the modest increase in cases of T1D. Reports from Italy and Canada in the early months of the pandemic showed a modest decrease in cases [1,4], while a German study found a nonsignificant increase that fell within the expected rate of annual increase in T1D [2]. These data were collected over the first several months of the pandemic and therefore do not accurately reflect the winter seasonality in the diagnosis of T1D [22]. Our finding of a slight increase in diagnoses of T1D during the pandemic is consistent with the German data and also with prior data showing an increasing incidence of T1D in the United States $[21,23]$. Interpretation of these changes is further complicated by more rapid rates of increase in T1D among NHB and Latinx youth who are well represented in our sample $[21,23]$.

Studies have posited that direct diabetogenic effects of SARS-CoV-2 may account for the increased incidence of diabetes during the pandemic. However, only $3.4 \%$ of all youth with new-onset diabetes tested positive, and infection rates were comparable among youth with T1D and T2D. Given marked differences in the rates of increase in T1D and T2D during the pandemic, it seems unlikely that direct effects of acute SARS-CoV-2 infection are solely responsible for the increase in new-onset diabetes. Because we collected only SARS-CoV-2 PCR tests and not 
antibodies, these data should be interpreted with caution as prior COVID-19 infections cannot be excluded. Furthermore, as pancreatic autoantibodies develop years before clinical symptoms of T1D, studies in the coming years should further explore a possible relationship between the SARS-CoV-2 virus and new-onset diabetes [24].

Before the pandemic, $59.6 \%$ of youth with new-onset T2D were female; however, during the pandemic, 58.9\% of youth were male. Pre-pandemic, T2D was female predominant in the United States [20], and male youth were known to engage in more moderate to vigorous physical activity than females [25]. During the COVID-19 pandemic, other studies have shown that screen time increased and physical activity decreased among youth of all ages and genders $[26,27]$. Levels of activity decreased the most among male adolescents, falling to levels comparable to those seen in females, which may explain these findings. We did not find any significant differences in weight or BMI changes preceding the diagnosis of T2D during the pandemic; however, data were underpowered and only available for $29.3 \%$ of youth. We did find a disproportionate increase in T2D among NHB youth who already accounted for $56.7 \%$ of cases pre-pandemic. During the pandemic, COVID-19-related school closures disproportionately affected minority youth of lower socioeconomic status, suggesting that indirect effects of social distancing measures may have contributed to the differences observed [28]. However, despite a similar prevalence of T2D among NHB and Latinx youth and adults $[23,29]$, there was not a clear increase in cases of T2D among Latinx youth during the pandemic.

Despite already high rates of DKA in our population, we found further increases during the pandemic. Before the pandemic, 30-34\% of American youth with new-onset T1D presented with DKA [20,30], whereas $41.4 \%$ and $51.9 \%$ of youth at our center presented with DKA in the 2 years before the pandemic. This finding likely represents the underserved population at our center as minority youth with T1D and those with public health insurance are at higher risk for DKA [20, 30, 31]. During the pandemic, overall rates of DKA did not change significantly in T1D. However, there was a significant $10 \% \mathrm{ab}-$ solute percentage point increase in severe DKA and more NHB youth presented in DKA during the pandemic. These findings are consistent with prior reports demonstrating increased rates of DKA among NHB people with known T1D during the pandemic [32]. These trends are worrisome given that $\mathrm{DKA}$ at diagnosis of $\mathrm{T} 1 \mathrm{D}$ is predictive of poor long-term glycemic control [33].
The 5.8\% incidence of DKA in youth with new-onset T2D pre-pandemic at our center was comparable to national studies [20]. While older studies showed a decreasing incidence of DKA in youth with new-onset T2D [20], during the pandemic, cases of DKA at our center increased nearly 6 -fold such that nearly one quarter of youth presented in DKA during the pandemic. This increases in DKA at diagnosis of T2D has also been reported in other urban pediatric diabetes centers [5]. Whereas, no child presented with hyperosmolar DKA pre-pandemic; $9.2 \%$ of youth with T2D had hyperosmolar DKA during the pandemic [15]. The incidence of hyperosmolar states has not been well studied, but the best available data suggest this condition occurs in $2-4 \%$ of youth with $\mathrm{T} 2 \mathrm{D}[34,35]$. This increase in severity of presentation is also reflected in an increase in the number of youth requiring inpatient admission at T2D diagnosis. Increased severity of presentation certainly contributed to the increase in admissions; however, it is possible that challenges in securing prompt outpatient endocrinology followup during the pandemic may have also contributed given the rapid increase in volume of urgent patients. More severe presentations of both T1D and T2D during the pandemic may reflect delays in seeking care, either due to lower rates of preventative care visits or possibly due to fear of seeking care in a hospital-based setting, reported in other studies [10, 11, 36, 37]. Increased psychological stress and subsequent elevations in cortisol levels during the pandemic may have also contributed to hyperglycemia and ketosis $[9,38]$.

Strengths of this study include our ability to capture all youth diagnosed with T1D and T2D at a highly diverse center in the 2 years before and the first 12 months of the COVID-19 pandemic. However, several important limitations require consideration. Because we were unable to measure the number of youth without diabetes in our catchment area, we could not calculate the incidence of diabetes. It is therefore possible that our findings reflect increased referrals to our center, though unlikely given declines in pediatric healthcare visits during the pandemic and decrease in the number of unique patients cared for at our center during the pandemic. Although chart review was conducted for youth not clearly meeting criteria for T1D or T2D, it is possible that some youth with monogenic diabetes may have been inadvertently captured. Results from SARS-CoV-2 PCR testing were available for most patients; however, the lack of screening in the outpatient setting and inability to collect SARS-CoV-2 antibodies may have led to an underestimation of the direct impact of the virus on our 
reported outcomes. Our ability to assess whether weight gain contributed to the increase in pediatric T2D in this retrospective cohort study was limited by an underpowered sample and possibly by interval weight loss from metabolic decompensation preceding the T2D diagnosis. Finally, this single-center study may not be generalizable to a larger population though the diversity of our population does shed light on staggering racial and ethnic differences.

In conclusion, rates of $\mathrm{T} 2 \mathrm{D}$ and the severity of presentation increased dramatically during the COVID-19 pandemic. As only a small percentage of youth tested SARS$\mathrm{CoV}-2$ positive at diagnosis, we suspect that indirect effects of social distancing measures and their disparate effects on minority and underserved communities explain these trends in T2D. Further studies seeking to better understand the root cause of the increase in rates of DKA and hyperosmolar DKA in T2D and increase in severity of DKA in T1D, which all disproportionately impacted NHB youth, are needed. Now, more than 1 year into the pandemic as we contemplate permanently incorporating pandemic-related lifestyle changes, such as teleworking, into our society, we must carefully consider the unintended, indirect effects of these measures and their potential for worsening long-term health consequences and pre-existing health disparities.

\section{Acknowledgments}

The authors wish to thank Jennifer Boughton, LCSW, and Alex Richardson, RD, CDCES, for their support in data gathering and categorization of types of insurance and Dr. Joseph Wolfsdorf and Dr. Stephanie Chung for their critical review of the manuscript.

\section{Statement of Ethics}

Study approval statement: this study protocol was reviewed and deemed exempt by institutional review board at the Children's National Hospital, decision number Pro0001519. Consent to participate: written informed consent from participants was not required in accordance with the committee decision.

\section{Conflict of Interest Statement}

B.E.M. has research support from Tandem, Dexcom, and the Cystic Fibrosis Foundation. M.M. is supported by a Pathway to Stop Diabetes Accelerator award from the American Diabetes Association (1-18-ACE-27). The other authors have conflicts of interest to disclosure.

\section{Funding Sources}

No funding was secured for this study.

\section{Author Contributions}

B.E.M., A.M., M.M., R.S., and E.E. formulated the clinical question. B.E.M. wrote the manuscript with contributions from M.E.F., and A.K., B.E.M., and J.G. completed the statistical analyses and created the figures. A.M., M.M., R.S., and E.E. made critical contributions to the manuscript. All the authors edited, reviewed, and approved the manuscript. B.E.M. is the guarantor of this work and, as such, had full access to all data in the study and takes responsibility for the integrity of the data and accuracy of the data analysis.

\section{Data Availability Statement}

All data generated or analyzed during this study are included in this article. Further inquiries can be directed to the corresponding author.

\section{References}

1 Rabbone I, Schiaffini R, Cherubini V, Maffeis C, Scaramuzza A. Has COVID-19 delayed the diagnosis and worsened the presentation of type 1 diabetes in children? Diabetes Care. 2020;43(11):2870-2.

2 Tittel SR, Rosenbauer J, Kamrath C, Ziegler J, Reschke F, Hammersen J, et al. Did the COVID-19 lockdown affect the incidence of pediatric type 1 diabetes in Germany? Diabetes Care. 2020;43(11):e172-3.

3 Dzygalo K, Nowaczyk J, Szwilling A, Kowalska A. Increased frequency of severe diabetic ketoacidosis at type 1 diabetes onset among children during COVID-19 pandemic lockdown: an observational cohort study. Pediatr Endocrinol Diabetes Metab. 2020;26(4):16775.
4 Ho J, Rosolowsky E, Pacaud D, Huang C, Lemay JA, Brockman N, et al. Diabetic ketoacidosis at type 1 diabetes diagnosis in children during the COVID-19 pandemic. Pediatr Diabetes. 2021;22(4):552-7.

5 Chao LC, Vidmar AP, Georgia S. Spike in diabetic ketoacidosis rates in pediatric type 2 diabetes during the COVID-19 pandemic. Diabetes Care. 2021.

6 Müller JA, Groß R, Conzelmann C, Krüger J, Merle U, Steinhart J, et al. SARS-CoV-2 infects and replicates in cells of the human endocrine and exocrine pancreas. Nat Metab. 2021;3(2):149-65.
7 Nogueira-de-Almeida CA, Del Ciampo LA, Ferraz IS, Del Ciampo IRL, Contini AA, Ued FDV. COVID-19 and obesity in childhood and adolescence: a clinical review. J Pediatr. 2020;96(5):546-58

8 Stojanovich L. Stress and autoimmunity. Autoimmun Rev. 2010;9(5):A271-6.

9 Rothe J, Buse J, Uhlmann A, Bluschke A, Roessner V. Changes in emotions and worries during the Covid-19 pandemic: an online-survey with children and adults with and without mental health conditions. Child Adolesc Psychiatry Ment Health. 2021; 15(1):11. 
10 Zhang H, Guo L-W, Gao Y-Y, Yao H, Xie $\mathrm{Z}-\mathrm{K}$, Zhang W-X. The impact of the COVID-19 pandemic on pediatric clinical practice in Wenzhou, China: a Retrospective Study. Front Pediatr. 2020;8.

11 Macy ML, Huetteman P, Kan K. Changes in primary care visits in the 24 weeks after COVID-19 stay-at-home orders relative to the comparable time period in 2019 in metropolitan Chicago and Northern Illinois. J Prim Care Community Health. 2020; 11: 2150132720969557.

12 American Diabetes Association. 2. Classification and diagnosis of diabetes: standards of medical care in diabetes-2021. Diabetes Care. 2021;44(Suppl 1):S15-33.

13 Kuczmarski RJ, Ogden CL, Guo SS, Grummer-Strawn LM, Flegal KM, Mei Z, et al. 2000 CDC Growth Charts for the United States: methods and development. Vital Health Stat 11. 2002;May;11(246):1-190.

14 Wolfsdorf JI, Glaser N, Agus M, Fritsch M, Hanas $\mathrm{R}$, Rewers A, et al. ISPAD clinical practice consensus guidelines 2018: diabetic ketoacidosis and the hyperglycemic hyperosmolar state. Pediatr Diabetes. 2018;19(Suppl 27):155-77.

15 Zeitler P, Haqq A, Rosenbloom A, Glaser N. Hyperglycemic hyperosmolar syndrome in children: pathophysiological considerations and suggested guidelines for treatment. J Pediatr. 2011;158(1):9-2.

16 Skinner AC, Skelton JA. Prevalence and trends in obesity and severe obesity among children in the United States, 1999-2012. JAMA Pediatr. 2014;168(6):561-6.

17 Flegal KM, Wei R, Ogden CL, Freedman DS, Johnson CL, Curtin LR. Characterizing extreme values of body mass index-for-age by using the 2000 Centers for Disease Control and Prevention growth charts. Am J Clin Nutr. 2009;90(5):1314-20.

18 Subcommittee on Standardized Collection of Race/Ethnicity Data for Healthcare Quality Improvement. In: Ulmer $\mathrm{C}$, McFadden $\mathrm{B}$, Nerenz DR, editors. Race, ethnicity, and language data: standardization for health care quality improvement. Washington, DC: $\mathrm{Na}-$ tional Academies Press; 2009.
19 Goyal MK, Simpson JN, Boyle MD, Badolato GM, Delaney M, McCarter R, et al. Racial and/or ethnic and socioeconomic disparities of SARS-CoV-2 infection among children. Pediatrics. 2020;146(4).

20 Dabelea D, Rewers A, Stafford JM, Standiford DA, Lawrence JM, Saydah S, et al. Trends in the prevalence of ketoacidosis at diabetes diagnosis: the SEARCH for diabetes in youth study. Pediatrics. 2014;133(4):e938-45.

21 Divers J, Mayer-Davis EJ, Lawrence JM, Isom S, Dabelea D, Dolan L, et al. Trends in incidence of type 1 and type 2 diabetes among youths: selected counties and Indian Reservations, United States, 2002-2015. MMWR Morb Mortal Wkly Rep. 2020;69(6):161-5.

22 Moltchanova EV, Schreier N, Lammi N, Karvonen M. Seasonal variation of diagnosis of Type 1 diabetes mellitus in children worldwide. Diabet Med. 2009;26(7):673-8.

23 Mayer-Davis EJ, Lawrence JM, Dabelea D, Divers J, Isom S, Dolan L, et al. Incidence trends of type 1 and type 2 diabetes among youths, 2002-2012. N Engl J Med. 2017; 376(15):1419-29.

24 Filippi CM, von Herrath MG. Viral trigger for type 1 diabetes: pros and cons. Diabetes. 2008; 57(11):2863-71.

25 Elhakeem A, Heron J, Tobias JH, Lawlor DA. Physical activity throughout adolescence and peak hip strength in young adults. JAMA Netw Open. 2020;3(8):e2013463.

26 Dunton GF, Do B, Wang SD. Early effects of the COVID-19 pandemic on physical activity and sedentary behavior in children living in the U.S. BMC Public Health. 2020;20(1):1351.

27 Tulchin-Francis K, Stevens WJr., Gu X, Zhang T, Roberts H, Keller J, et al. The impact of the coronavirus disease 2019 pandemic on physical activity in US children. J Sport Health Sci. 2021.

28 Parolin Z, Lee EK. Large socio-economic, geographic and demographic disparities exist in exposure to school closures. Nat Hum Behav. 2021
29 Centers for Disease Control and Prevention. National diabetes statistics report, 2020. Atlanta, GA; 2020.

30 Klingensmith GJ, Tamborlane WV, Wood J, Haller MJ, Silverstein J, Cengiz E, et al. Diabetic ketoacidosis at diabetes onset: still an all too common threat in youth. J Pediatr. 2013; 162(2):330-4.e1.

31 Everett EM, Copeland TP, Moin T, Wisk LE. National trends in pediatric admissions for diabetic ketoacidosis, 2006-2016. J Clin Endocrinol Metab. 2021;106(8):2343-54.

32 Ebekozien O, Agarwal S, Noor N, AlbaneseO'Neill A, Wong JC, Seeherunvong T, et al. Inequities in diabetic ketoacidosis among patients with type 1 diabetes and COVID-19: data from 52 US clinical centers. J Clin Endocrinol Metabol. 2020.

33 Duca LM, Wang B, Rewers M, Rewers A. Diabetic ketoacidosis at diagnosis of type 1 diabetes predicts poor long-term glycemic control. Diabetes Care. 2017;40(9):1249-55.

34 Fourtner SH, Weinzimer SA, Levitt Katz LE. Hyperglycemic hyperosmolar non-ketotic syndrome in children with type 2 diabetes*. Pediatr Diabetes. 2005;6:129-35.

35 Schmitt J, Rahman AF, Ashraf A. Concurrent diabetic ketoacidosis with hyperosmolality and/or severe hyperglycemia in youth with type 2 diabetes. Endocrinol Diabetes Metab. 2020;3(3):e00160.

36 Pines JM, Zocchi MS, Black BS, Carlson JN, Celedon P, Moghtaderi A, et al. Characterizing pediatric emergency department visits during the COVID-19 pandemic. Am J Emerg Med. 2021;41:201-4.

37 Santoli JM, Lindley MC, DeSilva MB, Kharbanda EO, Daley MF, Galloway L, et al. Effects of the COVID-19 pandemic on routine pediatric vaccine ordering and administration: United States, 2020. MMWR Morb Mortal Wkly Rep. 2020;69(19):591-3.

38 Linfoot P, Bergstrom C, Ipp E. Pathophysiology of ketoacidosis in Type 2 diabetes mellitus. Diabet Med. 2005;22(10):1414-9. 